Conflict of interest None.

Funding National institute for Health Research Collaboration for Leadership in Applied Health Research and Care East Midlands, UK.

\section{EXPERIENCE OF AMBULANCE WORKERS, NURSES AND DOCTORS OF HANDOVER OF PATIENTS WHO ARE TRANSPORTED BY AMBULANCES TO EMERGENCY DEPARTMENTS IN ICELAND: A QUALITATIVE INTERVIEW STUDY}

${ }^{1} S$ Dúason* ${ }^{1,2}$ B Gunnarsson, ${ }^{1} \mathrm{MH}$ Svavarsdóttir. ${ }^{1}$ University of Akureyri, Iceland; ${ }^{2}$ Akureyri Hospital, Iceland

\subsection{6/bmjopen-2019-EMS.31}

Background Adverse events healthcare are often caused by communication failure. Patient handover from ambulance personnel to specialized nurses and doctors in Emergency Departments carries the risk that that important information will be lost during the process, with consequences that may adversely affect patient well-being. The objective of this qualitative study was to analyze communication and transfer of responsibility during handover of patients arriving with ambulances in Emergency Departments in Iceland.

Method Vancouver school method of phenomenology was used. Participants were selected with a purpose sampling. Semi-structured individual interviews were conducted and supported by interview guide. The interviews were themed, followed by construction of an individual analysis model and overall analysis model.

Results A total of 17 ambulance workers, registered nurses and doctors described their experience of a patient handover in Emergency Department and the process of exchange of written and verbal information between health professionals involved in the handover of care. The main finding of the study was that structured communication and information disclosure have a great impact on the quality of patient handover. This is described in four main themes (Transfer of professional responsibility; Information dialogue; Personal and professional factors and Organizational factors) and nine sub-themes.

Conclusion Standardized handover protocol, clear procedures and education to healthcare professionals can potentially improve communication and transfer of responsibility for patients brought to emergency departments with ambulances, thus potentially improving patient safety.

Conflict of interest None.

Funding None.

\section{HELICOPTER EMERGENCY MEDICAL SERVICES MISSIONS TO SMALL ISLANDS AND THE MAINLAND DURING A 3-YEAR PERIOD IN DENMARK}

${ }^{1}$ TP Møller* ${ }^{2}$ AK Ersbøll, ${ }^{2}$ TM Kjærullf, ${ }^{2} \mathrm{~K}$ Birmann, ${ }^{3} \mathrm{~L}$ Knudsen, ${ }^{3} \mathrm{TM}$ Hansen, ${ }^{1} \mathrm{PA}$ Berlac, ${ }^{1} \mathrm{~F}$ Lippert, ${ }^{1} \mathrm{C}$ Barfod. ${ }^{1}$ Emergency Medical Services Copenhagen, University of Copenhagen, Denmark; ${ }^{2}$ National Institute of Public Health, Denmark; ${ }^{3}$ Helicopter Emergency Medical Services, Denmark

\subsection{6/bmjopen-2019-EMS.32}

Background The Danish Helicopter Emergency Medical Services (HEMS) is part of the Danish Emergency Medical Services System serving 5.7 million citizens with $1 \%$ living on small islands. HEMS is dispatched based on pre-defined criteria including severity and urgency of the case. HEMS is also sent to small islands for less urgent cases, when rapid transport is needed. The study aim was to characterize HEMS missions and the patient population focusing on differences in utilisation between small islands and mainland.

Method Descriptive study of data from the HEMS database in a three-year period from 1 October 2014 to 30 September 2017. All missions in which a patient was either treated on scene or transported by HEMS were included.

Results A total of 6551 HEMS missions were included in the study. Of those $986(15 \%)$ were missions to islands. In total, $92 \%$ of missions to islands resulted in patients being transported by HEMS compared to $66 \%$ of missions to mainland. Patients from islands were in general older than patients from the mainland. Disease severity, measured with National Advisory Committee for Aeronautics (NACA) score, was lower: 4-7 (serious or life-threatening conditions) in $41 \%$ and $66 \%$ of missions from islands and mainland, respectively.

Conclusion HEMS missions to islands counts for a substantial part of HEMS activity. The patient population is older and have lower disease severity. More insight into the patient population and outcomes after HEMS transportation would add to the planning and prioritizing of resources.

Conflict of interest None.

Funding The study was partly funded by The Danish HEMS.

\section{OHCA AND COPD, INCIDENCE, OUTCOME AND SEASONAL VARIATION}

${ }^{1}$ Lone Braagaard*, ${ }^{1,2}$ Theo W Jensen, ${ }^{2,3}$ Mathias G Holgersen, ${ }^{2} \mathrm{SN}$ Blomberg, ${ }^{2}$ Freddy Lippert, ${ }^{2}$ Helle C Christensen. ${ }^{1}$ Department of Pulmonary and Infectious Diseases, Nordsjaellands University Hospital, Hilleroed, Denmark; ${ }^{2}$ Emergency Medical Services Copenhagen, University of Copenhagen, Copenhagen, Denmark; ${ }^{3}$ Danish PCD and chILD Centre, CF Centre Copenhagen, Paediatric Pulmonary Service, Department of Paediatrics and Adolescent Medicine, Copenhagen University Hospital, Rigshospitalet, Denmark

\subsection{6/bmjopen-2019-EMS.33}

Background In Denmark, 30 day survival from OHCA have tripled from 2001-2011, but not among COPD patients. It is known that COPD patients are less likely to have; witnessed arrest, bystander actions and a shockable rhythm. The purpose of this study is to present updated rates of incident, survival to hospital, EMS treatment and seasonal variation among OHCA in COPD patients.

Method Data was collected from the verified 2016 Danish OHCA of patients with history of COPD noted in the EMS records. Incidence rates per 100.000 inhabitants, survival rates to hospital and EMS resuscitation attempt are presented. Fisher's exact and Chi-square test was used to assess significant differences in survival rates between COPD and non-COPD OHCA victims. Monthly variations of OHCA in COPD patients are presented as percentages.

Results In 2016 there were 1480 indexed and verified OHCA in the capital region of Denmark. Preliminary data show OHCA incidence rates in COPD patients is 8.4 per 100.000 inhabitants, survival to hospital rates of $29.1 \%$, and a seasonal variance with high incidences in winter months (range: $4 \%$ in April - $13.9 \%$ in November). There was a significant difference in termination of treatment prior to arrival at hospital (COPD: 69.5\%; non-COPD: 59.6\% p:0.004).

Conclusion Preliminary data show that COPD patients still have a significant worse outcome. The reported seasonal 
variance and incidence rates can aid campaigns to improve survival. Data extraction and analysis on treatment prior to arrest and clearing of confounding factors might lead to more effective campaigns to improve survival.

\section{REFERENCE}

1. Moeller, S.G. et al, Temporal trends in survival after out-of-hospital cardiac arrest in patients with and without underlying chronic obstructive pulmonary disease. Resuscitation 104 (2016) 76-82.

2. Karlsson, L.I.M. et al, Diurnal variations in incidence and outcome of out-of-hospital cardiac arrest including prior comorbidity and pharmacotherapy: A nationwide study in Denmark. Resuscitation 85 (2014) 1161-1168.

Conflict of interest None.

Funding None.

\section{EVALUATION OF DISPATCH OUTCOMES AND STAFFING OF THE COPENHAGEN MOBILE HEALTH AND SOCIAL CARE UNIT}

${ }^{1} \mathrm{SL}$ Østergaard*, 1,2RM Lyngby. ${ }^{1}$ Copenhagen Emergency Medical Services, Denmark; ${ }^{2}$ Kingston University and St George's, University of London, UK

\subsection{6/bmjopen-2019-EMS.34}

Background The mobile health-/social care unit (MHSCU) is a specialized unit within the Emergency Medical Services (EMS) in the Capital Region of Denmark. It provides acute social care for social deprived citizens and is staffed with a social worker and a paramedic. This study was to evaluate the MHSCU-dispatch outcomes and the combination of paramedical and social effort.

Method Data on the total number of MHSCU dispatches and outcomes in 2016 and 2017 was retrieved from the dispatch system operated by EMS Copenhagen and descriptively analyzed. Results MHSCU was dispatched 2976 times. Of these 384 patients $(12.9 \%)$ were brought to a somatic emergency department (ED) and $255(8.6 \%)$ to a psychiatric ED. A total of 355 patients (11.9\%) were left to selfcare while $196(6.6 \%)$ were brought to a shelter. Referred from EMS was $41(1.4 \%)$ and referred to EMS was $4(0.1 \%)$. referred to police was $13(0.4 \%)$. In 1386 (46.6\%) cases MHSCU were doing proactive work, citizen had left scene or MHSCU was cancelled. The remaining 342 (11.5\%) were 'unknown'.

Conclusion Based on the low referral to emergency ambulances and police, the dispatch of MHSCU seems well-prioritized. Dispatches-outcome also indicate the need for both paramedical and social staffing as there is an approximately even distribution between health related and social related referral. This study concludes that a MHSCU can serve as a valuable resource in EMS systems.

Conflict of interest None.

Funding None.

\section{USE OF MASS TRANSFUSION SCALES IN PREHOSPITAL SETTING}

${ }^{1}$ D Bélanger-Quintana*, 1J de la Cuerda del Olmo, ${ }^{1} E$ Sanz de Miguel, ${ }^{1} E$ Corral Torres, ${ }^{1} S$ Apilluelo Gutierrez, ${ }^{1} \mathrm{M}$ Castellanos Muñoz, ${ }^{1} \mathrm{~L}$ Ramirez Freire, ${ }^{1} \mathrm{LL}$ Zubillaga Carmona, ${ }^{2}$ B Bernardino Cuesta. ${ }^{1}$ SAMUR - Civil Protection, Madrid, SPAIN; ${ }^{2}$ Hospital Universitario Infantil Niño Jesus, Madrid, SPAIN

10.1136/bmjopen-2019-EMS.35
Background Hemorrhagic shock is one of the leading causes of death in trauma. Prompt implementation of transfusion with blood related products and surgery mitigate the effects of bleeding and shock. Early warning of mass transfusion needs by prehospital units could shorten the time of initiation of hospital protocols.

Objective To evaluate the adequacy of mass transfusion scales TASH and ABC in the prehospital setting.

Method Case control study of patients that were transfused in hospital and were treated and transported by SAMUR - Civil Protection. Both TASH and ABC scales were calculated and related with who received transfusion. Statistical analysis was used to describe the relation.

Results A total of 66 patients received transfusion. Median age of 41.5 years. $74.2 \%$ were male. A cut-off point of 16 or more was used as positive for transfusion need in the TASH scale and of 2 or more for the ABC scale. TASH showed a $100 \%$ sensitivity for transfusion need and $\mathrm{ABC}$ showed a $67 \%$. Conclusion Using predictive scales in the prehospital setting could give hospitals more time to activate their mass transfusion protocols. Scales should be easy to use and calculate. TASH has shown to be a good scale but is harder to calculate than ABC. Further testing of other scales could prove helpful to better the warning system between hospital and prehospital units.

\section{REFERENCES}

1. Massive transfusion predictive scores in trauma. Experience of a transfusion registry M. Chico-Fernández, C. García-Fuentes, M.A. Alonso-Fernández, D. Toral-Vázquez, S. Bermejo-Aznarez, E. Alted-López.

2. Trauma Associated Severe Hemorrhage (TASH)-Score: Probability of Mass Transfusion as Surrogate for Life Threatening Hemorrhage after Multiple Trauma Yücel, Nedim MD; Lefering, Rolf PhD; Maegele, Marc MD; Vorweg, Matthias MD; Tjardes, Thorsten MD; Ruchholtz, Steffen MD; Neugebauer, Edmund A. M. PhD; Wappler, Frank MD; Bouillon, Bertil MD; Rixen, Dieter MD; the 'Polytrauma Study Group of the German Trauma Society'.

3. Early prediction of massive transfusion in trauma: simple as $A B C$ (assessment of blood consumption)? Nunez TC1, Voskresensky IV, Dossett LA, Shinall R, Dutton WD, Cotton BA.

Conflict of interest None.

Funding None.

\section{DOES UNGUIDED CARDIO-PULMONARY-RESUSCITATION IN COPENHAGEN ACHIEVE HIGH QUALITY RECOMMENDATIONS?}

${ }^{1,2} \mathrm{RM}$ Lyngby*, ${ }^{2} \mathrm{~T}$ Quinn, ${ }^{2} \mathrm{D}$ Nikoletou, ${ }^{1} \mathrm{~F}$ Folke. ${ }^{1}$ Copenhagen Emergency Medical Services, Denmark; ${ }^{2}$ Kingston University and St George's, University of London, UK

10.1136/bmjopen-2019-EMS.36

Background Survival from out-of-hospital cardiac arrest (OHCA) is associated with the quality of cardio-pulmonaryresuscitation (CPR). The European Resuscitation Council (ERC) and American Heart Association (AHA) define high quality CPR as compression depth of 5-6 centimeters, compression rate of 100-120 compressions/minute, full recoil (>400 milliseconds) after each compression and a hands-on time (compression fraction) of at least 60\% (ERC) or $80 \%$ (AHA). The aim of this study was to investigate if unguided CPR performed by Copenhagen Emergency Medical Services (EMS) met these recommendations.

Method From October throughout December 2018, OHCA data were collected from ambulances within the Capital Region of Denmark using Zoll X-series defibrillator (without 